\title{
Quantitative Profile of the Uropathogenic Escherichia coli Outer Membrane Proteome during Growth in Human Urine ${ }^{\nabla}$
}

\author{
Christopher J. Alteri and Harry L. T. Mobley* \\ Department of Microbiology and Immunology, University of Michigan Medical School, \\ 5641 West Medical Center Drive, Ann Arbor, Michigan 48109
}

Received 12 January 2007/Returned for modification 26 February 2007/Accepted 27 March 2007

\begin{abstract}
Outer membrane proteins (OMPs) of microbial pathogens are critical components that mediate direct interactions between microbes and their surrounding environment. Consequently, the study of OMPs is integral to furthering the understanding of host-pathogen interactions and to identifying key targets for development of improved antimicrobial agents and vaccines. In this study, we used two-dimensional polyacrylamide gel electrophoresis (2D-PAGE) and tandem mass spectrometry to characterize the uropathogenic Escherichia coli (UPEC) outer membrane subproteome; 30 individual OMPs present on the bacterial surface during growth in human urine were identified. Fluorescence difference gel electrophoresis was used to identify quantitative changes in levels of UPEC strain CFT073 OMPs during growth in urine; six known receptors for iron compounds were induced in this environment, i.e., ChuA, IutA, FhuA, IroN, IreA, and Iha. A seventh putative iron compound receptor, encoded by CFT073 open reading frame (ORF) c2482, was also identified and found to be induced in urine. Further, the induction of these seven iron receptors in human urine and during defined iron limitation was verified by using quantitative real-time PCR (qPCR). An eighth iron receptor, fepA, displayed similar induction levels under these conditions as measured by qPCR but was not identified by 2D-PAGE. Addition of $10 \mu \mathrm{M} \mathrm{FeCl}_{2}$ to human urine repressed the transcription of all eight iron receptor genes. A number of fecal-commensal, intestinal pathogenic, and uropathogenic $E$. coli strains all displayed similar growth rates in human urine, showing that the ability to grow in urine per se is not a urovirulence trait. Thus, human urine is an iron-limiting environment and UPEC enriches its outer membrane with iron receptors to contend with this iron limitation.
\end{abstract}

The bacterial outer membrane represents the critical interface between the microorganism and its environment. For pathogenic bacteria, the proteins localized to the outer membrane directly interact with components of the host and can act as adhesins or receptors to facilitate colonization and mediate acquisition of nutrients and macromolecules from this restrictive niche $(6,26)$. Because these proteins are surface exposed, they are attractive targets for the development of improved antimicrobial agents and vaccines to eradicate infecting microorganisms or stimulate protective immune responses such as neutralizing or opsonizing immunoglobulins $(10,21)$. Elucidating the composition and profile of bacterial outer membrane proteins can help to better understand how specific bacterial pathogens interact with the host and to identify potential therapeutic targets.

Uropathogenic Escherichia coli (UPEC) bacteria are extraintestinal pathogens that cause the majority of uncomplicated urinary tract infections (UTI) (49). It has been determined that these infections impact public health by exacting more than two billion dollars of health care costs annually (7, 19). It is well known that UPEC bacteria utilize type 1 fimbriae ( fim) (5) and pyelonephritis-associated pili (pap) (34) to establish and maintain infection in the urinary tract. Additionally,

\footnotetext{
* Corresponding author. Mailing address: Department of Microbiology and Immunology, University of Michigan Medical School, 5641 Medical Science Building II, 1150 West Medical Center Drive, Ann Arbor, MI 48109. Phone: (734) 763-3531. Fax: (734) 764-3562. E-mail: hmobley@umich.edu.

${ }^{\nabla}$ Published ahead of print on 9 April 2007.
}

UPEC bacteria possess the ability to secrete toxins such as CNF1 (33), hemolysin $(48,51)$, and autotransporters $(11,12)$ to produce cytotoxic effects in the host and thus contribute to UPEC virulence. It has been shown that the murine urinary tract is an iron-limiting environment (42); consequently, UPEC bacteria contain multiple iron acquisition systems that allow the pathogen to survive and replicate within the urinary tract by scavenging iron molecules sequestered by the host $(27,36,40,45)$. Although much is known about how UPEC bacteria are able to cause UTI, very is little is known regarding the specific proteins and receptors within the outer membrane of these microorganisms during colonization of the urinary tract that may be exploited for the development of improved UTI therapies.

Bacterial membranes are complex permeability barriers composed mainly of phospholipids and lipopolysaccharides. Because the proteins associated with these lipid bilayers are hydrophobic and often contain multiple transmembrane domains, it has been difficult to solubilize and visualize the full complement of outer membrane proteins. Recent advances in proteomics technology have greatly improved the ability to analyze bacterial outer membrane proteins. Molloy and colleagues described the use of carbonate extraction and the strong detergent amidosulfobetaine-14 (ASB-14) to successfully visualize and identify $21(80 \%)$ of 26 of predicted $E$. coli K-12 outer membrane proteins (23). These techniques were used to characterize the Pseudomonas aeruginosa membrane subproteome (25) and to identify asymmetrically distributed membrane proteins in E. coli (16). The use of ASB-14 was also 
shown to increase the number of identified hydrophobic membrane proteins of Francisella tularensis (46).

Two-dimensional fluorescence difference gel electrophoresis (2D-DIGE) represents an important tool to monitor quantitative changes in proteomic profiles in response to specific environmental cues or genetic variation (47). 2D-DIGE utilizes advanced multiplexing cyanine dyes to fluorescently label proteins from two distinct populations. By using cyanine dyes with differing spectral properties ( $\mathrm{Cy} 2, \mathrm{Cy} 3$, and $\mathrm{Cy} 5)$, it is possible to compare changes in the level of proteins between two conditions or populations directly within the same gel, thus reducing the analytical gel-to-gel variation that has plagued comparative proteomics in the past (1). The use of a labeled internal standard representing equal amounts of proteins from the groups being examined allows the ratios of proteins to be normalized, standardized, and compared across multiple experiments, providing a means to accurately identify even modest changes in protein levels with high reproducibility and statistical confidence (18).

The application of 2D-DIGE to the study of microorganisms has proven to be useful for characterizing changes in proteomic profiles for bacteria in response to specific conditions or mutations. 2D-DIGE was used to identify significant changes in 179 protein spots for $E$. coli in response to treatment with benzoic acid, a common food preservative that reduces the proton-motive force across the membrane (52). By comparing biofilm bacteria to planktonic cells, it was determined that Streptococcus mutans upregulates the synthesis of glucan (a component of plaque) and competence proteins while downregulating nucleotide biosynthesis, glycolysis, and central metabolism during biofilm growth (30). 2D-DIGE was also used to identify novel Helicobacter pylori Cag-associated proteins by comparing the proteomic profiles of wild-type $\mathrm{Cag}^{+} \mathrm{H}$. pylori and a cag pathogenicity island deletion strain (4). It was also found by using 2D-DIGE that Staphylococcus aureus differentially expresses 120 proteins and coordinates multiple biochemical pathways in response to iron exposure changes and fur inactivation (8).

In this report, we describe the UPEC outer membrane subproteome during growth in human urine and the use of $2 \mathrm{D}$ DIGE to identify quantitative changes in the levels of carbonate-extracted outer membrane proteins. We also show that most $E$. coli strains demonstrate the ability to grow in human urine despite being classified as commensal, enteropathogen, or uropathogen and that UPEC simultaneously induces seven outer membrane receptors for iron in response to this environment. Using quantitative real-time PCR (qPCR), we were able to verify that the transcripts of the iron receptor identified by 2 D-DIGE were upregulated by UPEC growing in urine and during defined iron limitation. These findings demonstrate that human urine is an iron-limiting environment and UPEC compensates for this iron limitation by enriching its outer membrane with receptors for iron compounds.

\section{MATERIALS AND METHODS}

Bacteria. CFT073 is a prototypic UPEC strain isolated from the blood and urine of a patient with acute pyelonephritis (22); its genome has been sequenced and fully annotated (50). E. coli strains CFT108 and CFT142 were isolated from the blood and urine of pyelonephritis patients (22); strains F3, F11, and F39 were representative strains from a collection of 38 isolates from cystitis patients (44)
Strains EFC2, EFC9, and EFC25 are fecal-commensal organisms from a collection of 27 control strains of $E$. coli from fecal samples from healthy women (20 to 50 years old) who had not had symptomatic UTI, known bacteriuria, or antibiotics within 6 months of sampling (22). E. coli MG1655 (K-12) is a laboratory fecal-commensal strain. E. coli EDL933 (enterohemorrhagic) (32) and 2348/69 (enteropathogenic) (17) are wild-type enteric pathogens.

Culture conditions. Isolated colonies were used to inoculate overnight LuriaBertani (LB) medium cultures. Bacteria from overnight cultures were collected by centrifugation, washed with sterile phosphate-buffered saline, and used to inoculate prewarmed LB medium or human urine. For defined iron limitation, LB medium containing $10 \mathrm{mM}$ deferoxamine mesylate (Desferal; Sigma) was used as a growth medium. For human urine studies, midstream urine from 8 to 10 male and female donors was collected in sterile sample containers, pooled, and sterilized by vacuum filtration through a $0.22-\mu \mathrm{m}$-pore-size filter. Growth curves were established in triplicate by using a Bioscreen bioanalyzer in 0.4-ml volumes, and optical density at $600 \mathrm{~nm}$ was recorded every $15 \mathrm{~min}$. Growth curve experiments were also repeated in $50-\mathrm{ml}$ culture volumes contained within 250-ml Erlenmeyer flasks. For defined iron-replete culture conditions, pooled urine was supplemented with $10 \mu \mathrm{M} \mathrm{FeCl}_{2}$ (Sigma). All cultures were incubated at $37^{\circ} \mathrm{C}$, overnight $\mathrm{LB}$ medium cultures were grown with aeration, and urine cultures were grown statically. For the preparation of bacterial outer membranes, UPEC isolate CFT073 was grown statically to exponential phase (optical density at $600 \mathrm{~nm}=0.25$ ) in prewarmed $\mathrm{LB}$ medium or human urine at $37^{\circ} \mathrm{C}$ in five 100-ml cultures for each growth medium.

Preparation of outer membrane proteins. Bacteria were harvested from 500 $\mathrm{ml}$ of culture by centrifugation $\left(10,000 \times \mathrm{g}, 30 \mathrm{~min}, 4^{\circ} \mathrm{C}\right)$ and lysed in a French pressure cell at $20,000 \mathrm{lb} / \mathrm{in}^{2}$, and outer membrane proteins were prepared by carbonate extraction and ultracentrifugation modified from Molloy and colleagues (23). Briefly, harvested cells were washed and resuspended in $10 \mathrm{ml}$ of 10 mM HEPES, pH 7.0, containing $100 \mathrm{U}$ of Benzonase (Sigma). Following two passes through the chilled pressure cell, lysates were centrifuged $(7,500 \times g, 10$ min, $4^{\circ} \mathrm{C}$ ) to remove unbroken cells and the supernatants were diluted to $60 \mathrm{ml}$ with $0.1 \mathrm{M}$ carbonate buffer, $\mathrm{pH} 11.0$, and incubated with stirring at $4^{\circ} \mathrm{C}$ for $1 \mathrm{~h}$. The carbonate-extracted membranes were recovered by ultracentrifugation $\left(120,000 \times g, 1 \mathrm{~h}, 4^{\circ} \mathrm{C}\right)$, and the membrane pellets were rinsed with $10 \mathrm{mM}$ HEPES, pH 7.0, and solubilized in modified cell lysis buffer (30 mM Tris-Cl [pH 8.5], $2 \mathrm{M}$ thiourea, $7 \mathrm{M}$ urea, 1\% ASB-14) at room temperature for 30 to $60 \mathrm{~min}$. Following solubilization, membrane proteins were quantified by using the $2 \mathrm{D}$ Quant Kit (GE Healthcare) following the manufacturer's protocol and either immediately used in DIGE labeling procedures or stored at $-80^{\circ} \mathrm{C}$.

2D-DIGE and tandem mass spectrometry (MS/MS). For 2D-DIGE (47), bacterial proteins were minimally labeled with cyanine-derived fluors (CyDyes) containing an $N$-hydroxysuccinimide ester-reactive group as recommended by the manufacturer (GE Healthcare). To determine quantitative differences within the UPEC membrane proteome during growth in human urine, outer membrane proteins prepared from human urine cultures were labeled with $\mathrm{Cy} 3$, those prepared from LB broth were labeled with Cy5, and a pooled internal standard representing equal amounts of both urine and LB medium preparations was labeled with $\mathrm{Cy} 2$. For each labeling reaction, $50 \mu \mathrm{g}$ of protein was incubated with $400 \mathrm{pmol}$ CyDye for $30 \mathrm{~min}$. To stop the reaction, $1 \mu \mathrm{l}$ of $10 \mathrm{mM}$ lysine was added and the mixture was incubated for $10 \mathrm{~min}$. All labeling incubations were carried out on ice and protected from light. Following labeling, samples labeled with each CyDye were pooled ( $150 \mu \mathrm{g}$ total protein), mixed with an equal volume of $2 \times$ DIGE sample buffer $(7 \mathrm{M}$ urea, $2 \mathrm{M}$ thiourea, $10 \mathrm{mM}$ tributylphosphine [TBP; Sigma], $2 \times$ biolytes 3-10 [Bio-Rad], 2\% ASB-14), and incubated on ice for $10 \mathrm{~min}$. For rehydration, samples were brought to $0.35 \mathrm{ml}$ with $1 \times$ DIGE rehydration buffer ( $7 \mathrm{M}$ urea, $2 \mathrm{M}$ thiourea, $5 \mathrm{mM}$ TBP, $1 \times$ biolytes $3-10,1 \%$ ASB-14) and used to passively rehydrate pH 3 to 10 IPG strips (Bio-Rad) overnight at room temperature. Rehydrated IPG strips were equilibrated (23) and subjected to isoelectric focusing for $40,000 \mathrm{~V} \cdot \mathrm{h}$ and second-dimension sodium dodecyl sulfate-polyacrylamide gel electrophoresis (SDS-PAGE) on 10\% gels (23) within low-fluorescence glass plates (Jule Biotechnologies, Inc.) and were run at a constant current of $55 \mathrm{~mA}$ at $4^{\circ} \mathrm{C}$ for $4 \mathrm{~h}$. Following SDS-PAGE, images were acquired and pixel intensity was obtained by using a Typhoon scanner (GE Healthcare) and differential in-gel analysis and biological analysis of variance were performed by using the DeCyder 6.5 software suite (GE Healthcare). With this software, the normalized spot volume ratios from $\mathrm{Cy} 3$ or Cy5 labeled spots were quantified relative to the Cy2-labeled internal standard from the same gel. The Cy2-labeled standard was then used to standardize and compare normalized volume ratios of the Cy3-and Cy5-labeled proteins between gels representing three independent experiments to generate statistical confidence for abundance changes by using Student's $t$ test and analysis of variance. To identify the proteins, $350 \mu \mathrm{g}$ of outer membrane proteins was focused as de- 
TABLE 1. Oligonucleotide primers for quantitative real-time PCR

\begin{tabular}{|c|c|c|c|}
\hline \multirow{2}{*}{ Gene } & \multirow{2}{*}{ ORF } & \multicolumn{2}{|c|}{ Sequence $^{a}$} \\
\hline & & Forward & Reverse \\
\hline gарA & c2184 & AAGTTGGTGTTGACGTTGTCGC & AGCGCCTTTAACGAACATCG \\
\hline btuB & c4929 & TTGCGCCAACTACTGTTGTGAC & ACATGACTGGCATTTGTACCGC \\
\hline$T s x$ & c0521 & ACGACAAACCGCAGTATCTTTCCG & AGCGAATGCTTCGTACTCAAGG \\
\hline tolC & c3781 & AATGAAGCGCGCAGTCCATTAC & TTAACGCACGCCATTTCGAC \\
\hline $\operatorname{ton} B$ & c1717 & TGCATTCATGGTGCTGTTGTGG & TTGTGGCGGTTCAAGATCAG \\
\hline $\operatorname{irg} A$ & c5174 & TCCTGTGGAAGCAATTGAACGC & AAGTACGCCATCCCATGTTTGG \\
\hline iha & c3610 & TGCGAATAACCACTCTGGCTTC & TAATCACAGAAACACTGGCGGC \\
\hline $\mathrm{NA}^{b}$ & $\mathrm{c} 2482$ & ATCGTTCGGCAAGCAACCTTTG & ATGCGGATTTGTTTACGGCCTG \\
\hline iroN & $\mathrm{c} 1250$ & ATTACCAAACGTCCCACCAACG & AAACGCGTGGTAAGAGCATCAC \\
\hline fhuA & $\mathrm{c} 0185$ & ACGGCCAAAGCCAGAATAAC & TTACCGTAAAGCACGGAAACCG \\
\hline fерA & c0669 & ATTGATATTCGCGGCATGGGTC & ACGTTCAATCATTTCAGGCGGC \\
\hline iut $A$ & c3632 & AAAGAGCTGAAAGACGCACTGG & TGTCGGAACGTGAAGAGTTGAG \\
\hline chuA & c4308 & AGCGTGTTGAGATTGTTCGC & AAACCACTGCTTTGTCCTTCCTGC \\
\hline $\operatorname{cir} A$ & c2690 & AAAGAAGTGCCTGGCGTACAAC & AAATCATTGTGGCGGAAGACGG \\
\hline
\end{tabular}

${ }^{a}$ Primer sequences are listed from $5^{\prime}$ to $3^{\prime}$.

${ }^{b}$ NA, no gene name available.

scribed above and the spots of interest were excised from a colloidal Coomassiestained 2D SDS-PAGE gel and subjected to enzymatic digestion with trypsin. Mass spectra were acquired on an Applied Biosystems 4700 Proteomics Analyzer (TOF/TOF). MS spectra were acquired from 800 to 3,500 Da, and the eight most intense peaks in each MS spectrum were selected for MS/MS analysis. Peptide identifications were obtained by using GPS Explorer (v3.0; Applied Biosystems), which utilizes the MASCOT search engine. Each MS/MS spectrum was searched against NCBInr. Tryptic digestion and MS/MS were performed at the University of Michigan Proteome Consortium.

RNA extraction and qPCR. For the extraction of total RNA, $1 \mathrm{ml}$ of bacteria culture was added to $0.125 \mathrm{ml}$ of ice-cold phenol-ethanol stop solution (5\% phenol in ethanol), and the bacteria were collected by centrifugation. Cells were lysed and RNA was extracted by using the RNeasy kit (QIAGEN) and following the manufacturer's recommended protocol. Following elution, nucleic acid concentrations were determined by spectrophotometry (NanoDrop) and residual DNA contamination was removed by incubating the samples with $4 \mathrm{U}$ of TURBO DNase (Ambion). After DNase inactivation, the RNA was recovered, quantified, and used as a template for PCR to confirm inactivation of contaminating DNA. The PCR-negative RNA was used for first-strand cDNA synthesis by using SuperScript II reverse transcriptase (Invitrogen). For cDNA synthesis, the manufacturer's protocol was followed, starting with $1.35 \mu \mathrm{g}$ total RNA template and $50 \mathrm{ng}$ random hexamers. Following synthesis, the cDNA was purified on a QIAquick column (QIAGEN), quantified by using the NanoDrop, and diluted to $5 \mathrm{ng} / \mu \mathrm{l}$ with nuclease-free water. RNA transcripts were quantified on an MX3000P real-time PCR machine (Stratagene) by using Brilliant SYBR green QPCR mix (Stratagene) in $25-\mu$ l volumes containing $25 \mathrm{ng}$ cDNA. Optimal primer concentrations were determined empirically. Primer sequences are listed in Table 1. For comparative, quantitative analysis, transcript levels were normalized to the level of gapA (glyceraldehyde 3-phosphate dehydrogenase A) and changes were determined by using an experiment-specific calibrator (LB medium) and the MXPro v 3.00 software package (Stratagene). For iron-replete qPCR, pooled urine was used as the calibrator to monitor transcript levels during growth in urine supplemented with iron.

\section{RESULTS}

Growth of $\boldsymbol{E}$. coli strains in human urine. It has been suggested that growth in urine may represent a feature restricted to uropathogenic bacteria or asymptomatic bacteriuria strains $(2,35,38,43)$. To determine whether the capacity to grow in human urine is restricted to UPEC, we analyzed growth rates for 12 fecal-commensal, uropathogenic, and enteropathogenic strains cultured in human urine (Fig. 1). Surprisingly, it was found that all but one of the E. coli strains examined possessed remarkably similar growth rates during growth in human urine (Fig. 1A). Even enteropathogenic and enterohemorrhagic $E$. coli strains produced growth curves indistinguishable from those of uropathogenic isolates. Similarly, three of four fecalcommensal E. coli strains grew as well as uropathogenic strains (Fig. 1A). E. coli strain MG1655 (K-12) was the only exception in these experiments, where K-12 displayed a significantly increased lag phase of $8.43 \pm 0.68$ h relative to CFT073 $(P=$ 0.0003 ) (Fig. 1B) under defined inoculation conditions. Despite this increased lag phase, E. coli $\mathrm{K}-12$ was able to grow
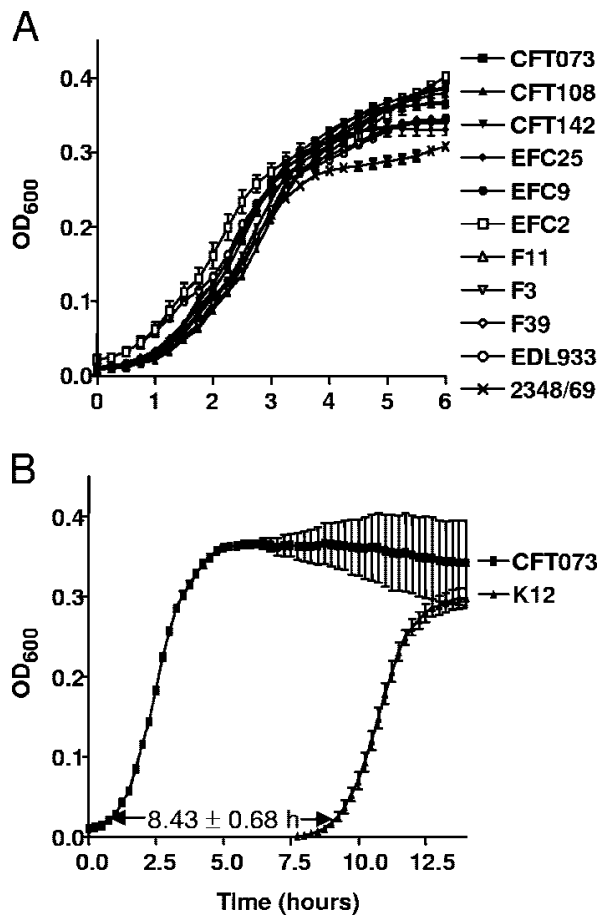

FIG. 1. Growth of uropathogenic, diarrheagenic, and fecal-commensal E. coli isolates cultured in human urine. (A) Growth profile for pyelonephritis, fecal-commensal, cystitis, enterohemorrhagic, and enteropathogenic strains during static culture at $37^{\circ} \mathrm{C}$ in sterilized urine pooled from 10 male and female donors. (B) Comparison of growth characteristics of E. coli CFT073 (UPEC) and MG1655 (K-12) in pooled human urine. $\mathrm{OD}_{600}$, optical density at $600 \mathrm{~nm}$. 
$\mathrm{pH} 3$

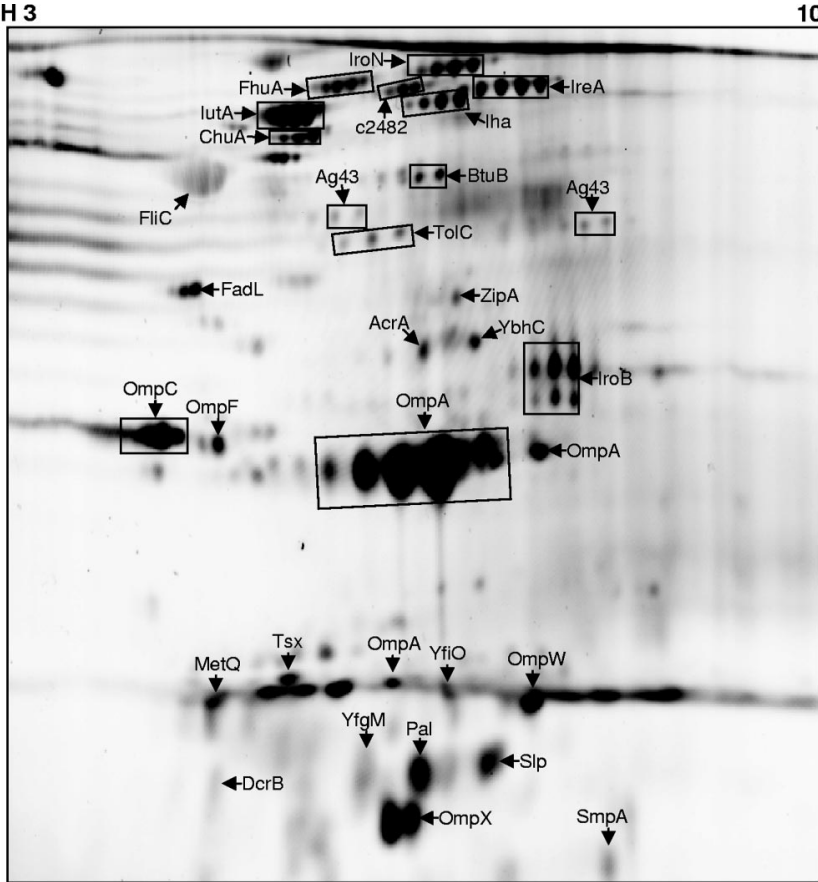

FIG. 2. Outer membrane subproteome for UPEC during growth in human urine. 2D gel of carbonate-insoluble membrane proteins prepared from CFT073 following culture in urine. Outer membrane fraction $(150 \mu \mathrm{g}$ protein) was isoelectrically focused on a pH 3 to 10 IPG strip, followed by $10 \%$ SDS-PAGE. The image shows the proteins identified by MS/MS (arrows). Boxed areas indicate proteins where multiple isoforms produced the same identification. The CFT073 annotated gene numbers, theoretical masses, and isoelectric points of the identified proteins are listed in Table 2. logarithmically at a rate indistinguishable from that of the other strains examined (Fig. 1B). These data demonstrate that the ability to grow in human urine is not limited to uropathogenic bacteria and do not explain selective colonization of the urinary tract by UPEC strains. Despite this finding, human urine has been a useful tool to identify UPEC genes that contribute to virulence $(36,37)$.

UPEC outer membrane subproteome during growth in urine. Outer membrane proteins represent important targets for the development of antimicrobial agents and vaccines partly because they are surface exposed. Complete solubilization of membrane proteins is critical for proteomic analysis of membrane fractions. The carbonate extraction method of preparing bacterial membranes combined with solutions containing detergents and reducing agents such as ASB-14 and TBP has vastly improved the visualization of membrane proteins $(23,24)$. By using these techniques, it was possible for us to resolve 104 protein isoforms and identify 30 unique outer membrane proteins from UPEC cultured in urine (Fig. 2 and Table 2). These include the porins OmpA, OmpC, OmpF, OmpW, and OmpX, in addition to the outer membrane assembly complex proteins YfiO and YfgM. Six outer membrane lipoproteins, SmpA, MetQ, Slp, Pal, FadL, and YbhC, were also identified. TolC and 10 putative and known TonB-dependent receptors also localized to the outer membrane in our experiments (Fig. 2 and Table 2). These data show that we were able to efficiently isolate, solubilize, and visualize the UPEC outer membrane subproteome for subsequent quantitative analysis.

TABLE 2. UPEC outer membrane subproteome following culture in urine

\begin{tabular}{|c|c|c|c|c|}
\hline Identification & ORF & Function & $M_{\mathrm{r}}^{a}$ & $\mathrm{pI}^{a}$ \\
\hline IutA & $\mathrm{c} 3623$ & Ferric aerobactin receptor & 84,050 & 5.24 \\
\hline ChuA & c4308 & Heme/hemoglobin receptor & 71,100 & 5.02 \\
\hline $\mathrm{NA}^{b}$ & c2482 & Putative receptor for iron compound & 79,178 & 5.42 \\
\hline IroB & $\mathrm{c} 1254$ & Glucosyltransferase & 42,306 & 6.06 \\
\hline FhuA & $\mathrm{c} 0185$ & Ferrochrome-iron receptor & 82,182 & 5.33 \\
\hline Iha & $\mathrm{c} 3610$ & Iron-regulated adhesin, catecholate receptor & 76,511 & 5.64 \\
\hline IroN & c1250 & Siderophore receptor & 79,385 & 5.79 \\
\hline IreA & c5174 & Iron-regulated virulence protein & 75,306 & 6.15 \\
\hline MetQ & $\mathrm{c} 0238$ & D-Methionine binding lipoprotein & 29,318 & 5.13 \\
\hline $\mathrm{OmpF}$ & c1071 & Porin & 39,354 & 4.78 \\
\hline TolC & c3781 & Outer membrane efflux protein & 55,053 & 5.80 \\
\hline OmpA & c1093 & Porin & 41,054 & 6.24 \\
\hline OmpC & c2758 & Porin & 41,225 & 4.59 \\
\hline Slp & $\mathrm{c} 4304$ & Starvation-inducible outer membrane lipoprotein & 22,210 & 6.27 \\
\hline YbhC & c0849 & Pectin methylesterase lipoprotein & 67,075 & 7.00 \\
\hline HemX & $\mathrm{c} 4722$ & Putative uroporphyrin methyltransferase & 43,957 & 4.61 \\
\hline BtuB & c4929 & Vitamin $\mathrm{B}_{12}$ (cobalamin) receptor & 68,397 & 5.42 \\
\hline SmpA & c3139 & Small protein A (outer membrane lipoprotein) & 12,302 & 8.71 \\
\hline OmpW & $\mathrm{c} 1722$ & Pole-localized outer membrane protein & 25,877 & 5.93 \\
\hline Tsx & $\mathrm{c} 0521$ & Nucleoside-specific pore-forming protein & 35,480 & 5.82 \\
\hline FadL & c2889 & Outer membrane long-chain fatty acid transport protein & 48,680 & 4.80 \\
\hline $\operatorname{Ag} 43$ & c1273 & Type V secretion/autotransporter, adhesin & 113,152 & 6.20 \\
\hline AcrA & $\mathrm{c} 0581$ & Acriflavine resistance and efflux protein & 43,521 & 8.41 \\
\hline ZipA & c2946 & Membrane-tethered cell division protein & 36,967 & 5.63 \\
\hline YfgM & $\mathrm{c} 3035$ & Membrane protein assembly complex & 22,146 & 5.07 \\
\hline YfiO & $\mathrm{c} 3117$ & Essential outer membrane lipoprotein & 27,829 & 6.16 \\
\hline OmpX & $\mathrm{c} 0900$ & Acid- or base-induced putative adhesin & 18,603 & 6.56 \\
\hline DcrB & $\mathrm{c} 4265$ & Outer membrane phage adsorption protein & 22,160 & 6.11 \\
\hline FliC & c2338 & Flagellin & 60,938 & 4.68 \\
\hline
\end{tabular}

${ }^{a}$ Theoretical masses and isoelectric points were determined by using the ExPASy proteomics server UniProt Knowledgebase (http://ca.expasy.org).

${ }^{b}$ NA, no gene name available. 


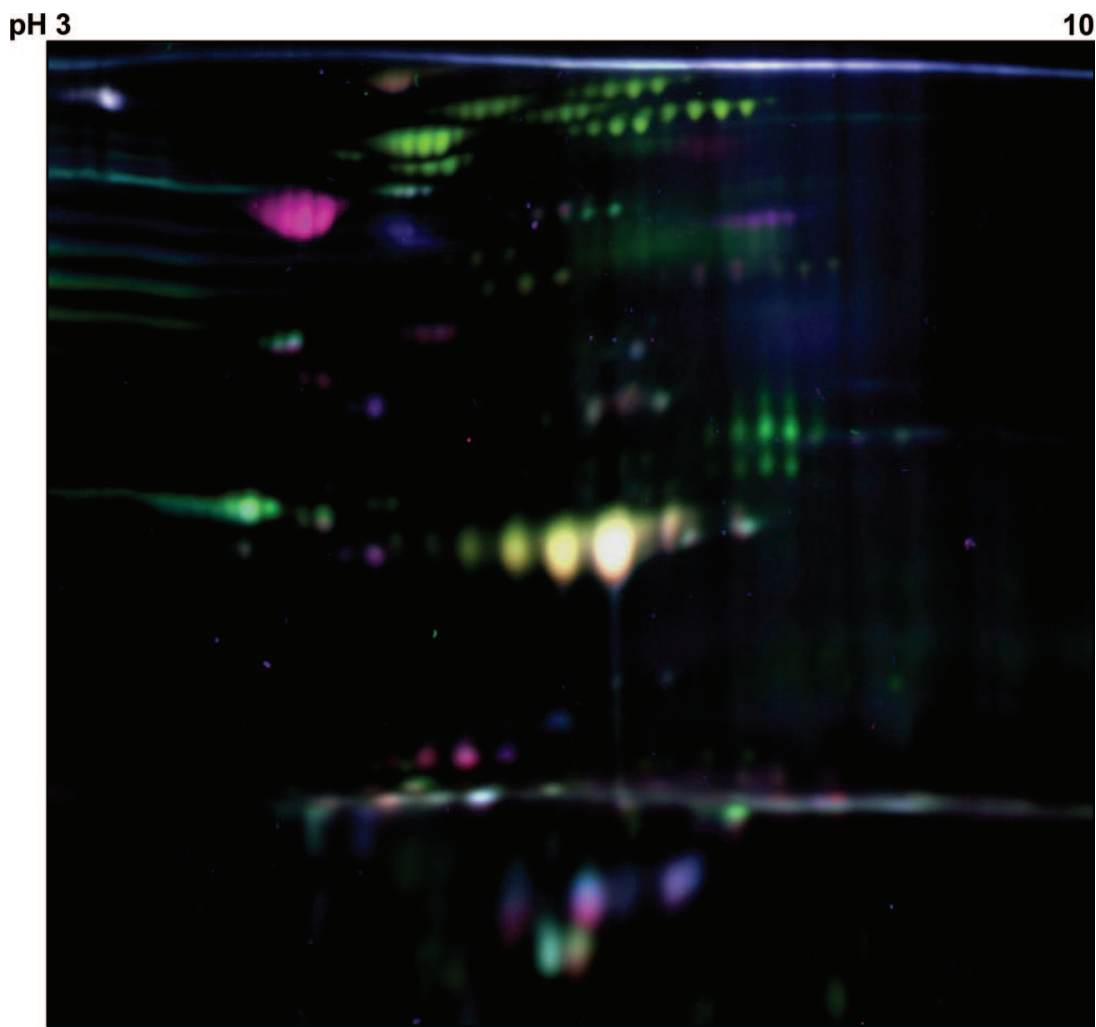

FIG. 3. 2D-DIGE of UPEC outer membrane proteins during growth in urine. Outer membrane proteins $(50 \mu \mathrm{g})$ from $E$. coli CFT073 cultured in urine were labeled with Cy3 (green), those from CFT073 grown in LB medium were labeled with Cy5 (red), and the pooled internal standard representing equal amounts of urine and LB medium outer membrane proteins was labeled with Cy2 (blue). The labeled proteins, $150 \mu \mathrm{g}$, were pooled and applied to a $\mathrm{pH} 3$ to 10 IPG strip and second-dimension 10\% SDS-PAGE. Green spots indicate protein features induced in urine, red spots represent proteins induced in LB medium, and blue spots show proteins that are similarly expressed in both urine and LB medium.

2D-DIGE. We used 2D-DIGE to determine quantitative differences in UPEC outer membrane proteins that are differentially expressed between growth in human urine and growth in LB medium. By multiplexing protein samples labeled with cyanine dyes, it is possible to compare the spot volumes between two conditions within the same gel, thus avoiding analytical gel-to-gel variation (1). Within a single gel, quantitative determinations can be made relative to the internal standard that includes an equal amount of proteins from each of the conditions being investigated (18). For our studies, we minimally labeled outer membrane proteins isolated from UPEC grown in urine with Cy3 (green), those from UPEC grown in LB medium with Cy5 (red), and the internal standard containing an equal amount of both outer membrane protein populations with $\mathrm{Cy} 2$ (blue). These proteins, covalently modified with the fluorescent dyes, were applied to the same 2D PAGE gel so that the relative amount of protein in each spot could be determined by analyzing the fluorescence spectrum of each dye. A representative 2D-DIGE gel image of E. coli CFT073 outer membrane proteins overlaid with three channels representing the multiplexed samples is shown in (Fig. 3), where the green spots represent protein isoforms induced in urine, red spots are proteins expressed more in LB medium, and blue spots are proteins similarly expressed in urine and LB medium.

Deconvolution of the spectral data by using differential ingel analysis software allows comparison of the relative abun- dance of the outer membrane proteins to be compared between the two populations. The Cy3 and Cy5 pixel intensities for identical spot boundaries are normalized by using the internal standard, and the resulting spot volumes can be viewed as three-dimensional (3D) peaks, as shown in Fig. 4. For each section of the DIGE scan containing the identified protein spots (rectangles in Fig. 4), the 3D view reflects the relative amount of each outer membrane protein for UPEC strain CFT073 cultured in urine or LB medium (Fig. 4). By comparing average changes in spot volumes across triplicate independent outer membrane preparations, statistical confidence in the relative abundance change for each spot was assigned by using the Student $t$ test (Fig. 5 and Table 3).

One class of outer membrane proteins significantly upregulated $(P<0.007)$ in human urine are seven putative and known TonB-dependent iron compound receptors (Fig. 4A and Table 3 ). These include the $>5$-fold induction (5.87- to 49.24-fold) of the known siderophore receptors IutA (aerobactin), FhuA (ferrochrome), and IroN (salmochelin) and the IrgA homolog adhesin Iha (enterobactin) (Fig. 5). Also reflecting the importance of iron acquisition in urine, there were a 28 -fold increase in the level of the heme receptor ChuA $(P=0.00045)$ and an 8 -fold increase in another IrgA homolog known as the ironresponsive element IreA in human urine $(P=0.00017)$ (Fig. 5 and Table 3). Interestingly, we found that a newly identified outer membrane antigen of CFT073, annotated as c2482 (E. C. 


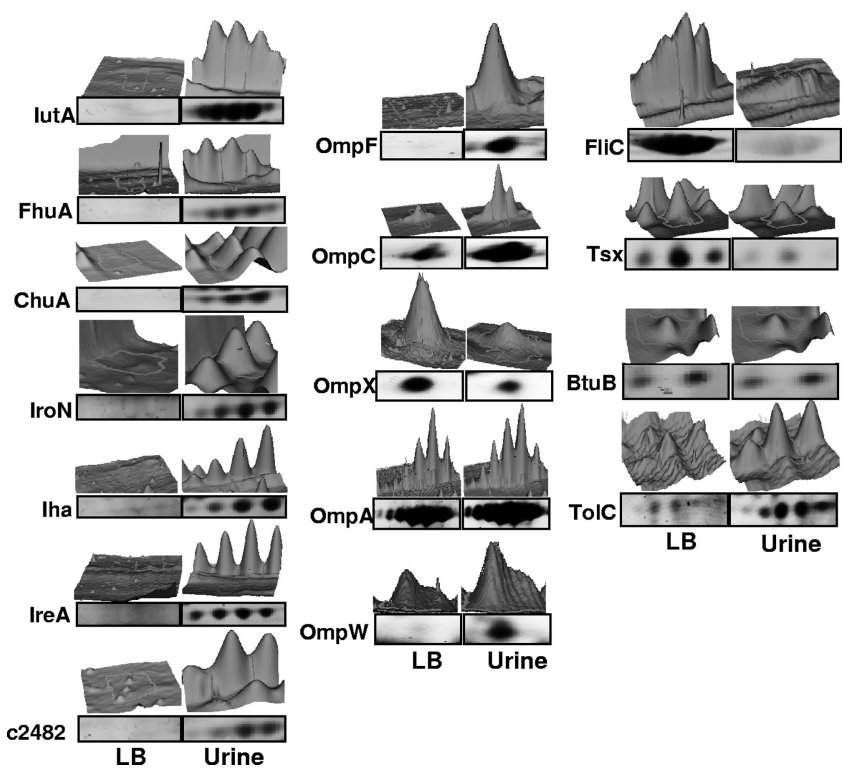

FIG. 4. Normalized spot volumes from multivariable 2D-DIGE scan of urine (Cy3) and LB medium (Cy5) UPEC outer membrane proteins. (A) Spot volumes for known and predicted CFT073 TonBdependent outer membrane receptors for iron, (B) outer membrane porins, and $(\mathrm{C})$ proteins showing decreased expression in urine or similar expression patterns in LB medium and urine. The 3D peaks represent the normalized pixel intensities for identical spot boundaries between the Cy3 and Cy5 scans. The boxed regions below the peaks are the corresponding protein isoforms present in the DIGE scan.
TABLE 3. Outer membrane proteins differentially expressed in urine

\begin{tabular}{lcl}
\hline Protein & $n$-Fold change $^{a}$ & $P$ value $^{b}$ \\
\hline IutA & 49.24 & 0.00004 \\
ChuA & 27.81 & 0.00045 \\
c2482 & 14.82 & 0.0011 \\
IrgA-like & 7.81 & 0.00017 \\
IroN & 7.63 & 0.0043 \\
FhuA & 7.25 & 0.0011 \\
Iha & 5.87 & 0.0071 \\
Tsx & 2.87 & 0.0065 \\
OmpA & 1.83 & 0.014 \\
BtuB & 1.56 & 0.032 \\
FadL & -4.23 & 0.0058 \\
FliC & -5.77 & 0.0012 \\
\hline${ }^{a} n$-Fold change relative to protein expression in LB medium. & \\
${ }^{b}$ Average $n$-fold change compared by using the Student $t$ test. &
\end{tabular}

Hagan and H. L. T. Mobley, unpublished data) that has homology to other TonB-dependent iron receptors, was induced 15 -fold during growth in human urine compared to LB medium culture $(P=0.0011)$ (Fig. 5 and Table 3$)$. Of all of these iron compound receptors, the aerobactin receptor IutA was induced to the highest level (49-fold) in human urine $(P<$ 0.0001) (Fig. 5 and Table 3).

Many of the porins displayed apparent differential expression between urine and LB medium cultures in representative 2D-DIGE experiments (Fig. 4B). Among these were the reciprocally regulated passive diffusion pores $\mathrm{OmpC}$ and $\mathrm{OmpF}$ and the integral outer membrane proteins $\mathrm{OmpX}$ and $\mathrm{OmpW}$. $\mathrm{OmpW}, \mathrm{OmpC}$, and $\mathrm{OmpF}$ appear to be expressed relatively
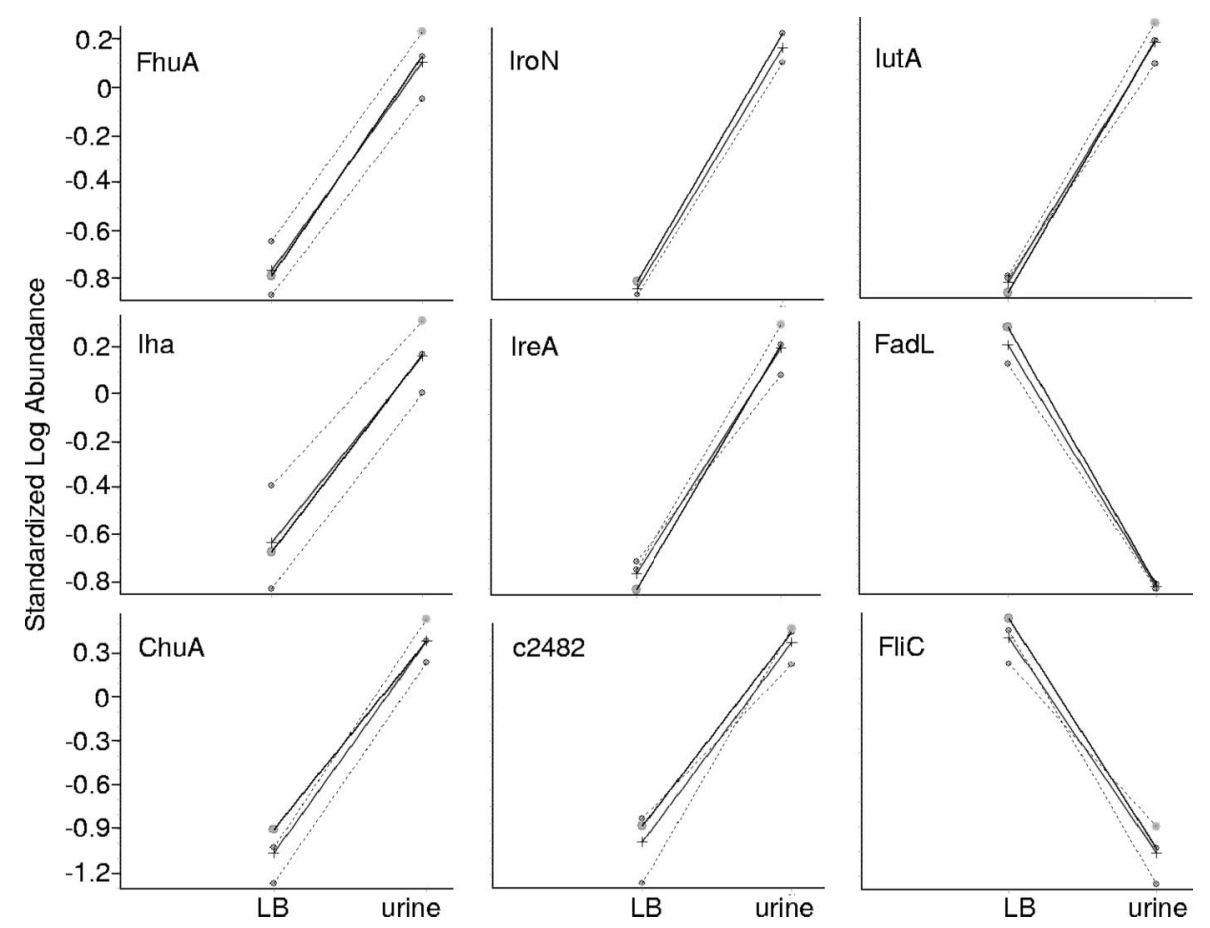

FIG. 5. 2D-DIGE expression patterns for UPEC outer membrane proteins differentially expressed in urine and LB medium. The standardized $\log _{10}$ abundance from triplicate 2D-DIGE experiments was generated by using the internal standard included in every gel. The Cy2 internal standard signals were used to normalize the ratios of LB to urine across multiple gels. The average abundance (solid lines) and individual quantitative changes (dashed lines) between normalized Cy3 (urine) and Cy5 (LB medium) signals are shown. 
more highly in urine than in LB medium, while OmpX expression appeared higher in LB medium (Fig. 4B); however, we were unable to assign significance to these changes. Despite the presence of at least eight isoforms of OmpA (Fig. 2), it was possible to visualize a modest 1.83 -fold induction of this porin in human urine $(P=0.014)$ (Fig. $4 \mathrm{~B}$ and Table 3$)$. Taken together, these data suggest that there exists considerable heterogeneity among the expression profiles for the outer membrane porins of UPEC between growth in urine and that in LB medium.

Other outer membrane proteins of UPEC that were identified in the membrane subproteome and 2D-DIGE are the TonB-dependent receptors BtuB and Tsx, the efflux protein TolC, the fatty acid-scavenging lipoprotein FadL, and the flagellin FliC (Fig. 4C). BtuB, a receptor that binds cobalamin (vitamin $\mathrm{B}_{12}$ ), and Tsx, which is involved in the import of nucleotides, were modestly induced during growth in human urine, 1.56- and 2.87-fold, respectively $(P<0.05)$ (Fig. 4C and Table 3). This is in contrast to FadL, which is downregulated 4.23-fold in urine compared to LB medium $(P=0.0058)$, and Fli, which is downregulated 5.77-fold $(P=0.0012)$ (Fig. 5 and Table 3). In summary, the 2D-DIGE analysis shows that UPEC highly induces the expression of multiple TonB-dependent iron compound receptors in urine, while other TonBdependent receptors such as BtuB and Tsx are only modestly induced. Interestingly, only two proteins, $\mathrm{FadL}$ and $\mathrm{FliC}$, were identified as being significantly downregulated in human urine, suggesting that fatty acid import and motility are likely not required for UPEC during growth in human urine.

Transcriptional analysis of iron receptor genes. Since the levels of seven TonB-dependent outer membrane iron receptor proteins were found to be induced by UPEC during growth in urine, the transcription of the genes that encode them should also be upregulated by UPEC in this environment. To confirm the 2D-DIGE findings, we used qPCR to compare the levels of CFT073 transcripts between urine and LB medium for ire A, iha, c2482, iroN, fhuA, iutA, chuA, tolC, tsx, and butB (Fig. $6 \mathrm{~A}$ ). Consistent with the 2D-DIGE results, iut $A$ was the most highly induced gene, displaying a 273-fold induction compared to LB medium (Fig. 6A). Of the iron receptors identified by 2D-DIGE, ChuA was the second most highly induced protein, and in our qPCR experiments we found the chuA transcript to be induced 206-fold versus its expression level in LB medium. Similarly, c2482, was induced 190-fold in urine and iroN displayed a 127 -fold induction (Fig. 6A). All of the remaining iron receptors identified as increased in urine by 2D-DIGE, ire $A$, $i h a$, and $f h u A$, also demonstrated induction in urine at the level of transcription by qPCR (Fig. 6A). Interestingly, FepA was not identified in the UPEC urine outer membrane subproteome or by 2D-DIGE but was induced 232 -fold in urine as measured by qPCR (Fig. 6A). Although BtuB and Tsx were modestly induced in human urine, as determined by 2D-DIGE, it was found that their transcripts were downregulated in these qPCR experiments (Fig. 6A). In general, these data show considerable agreement between the levels of induction seen by 2D-DIGE and qPCR; especially for the iron receptors; however, the qPCR data for $b t u B$ and $t s x$ suggest that mRNA and protein stability may affect the correlation between proteomic and transcriptional profiling.

To directly test whether the observed induction of UPEC
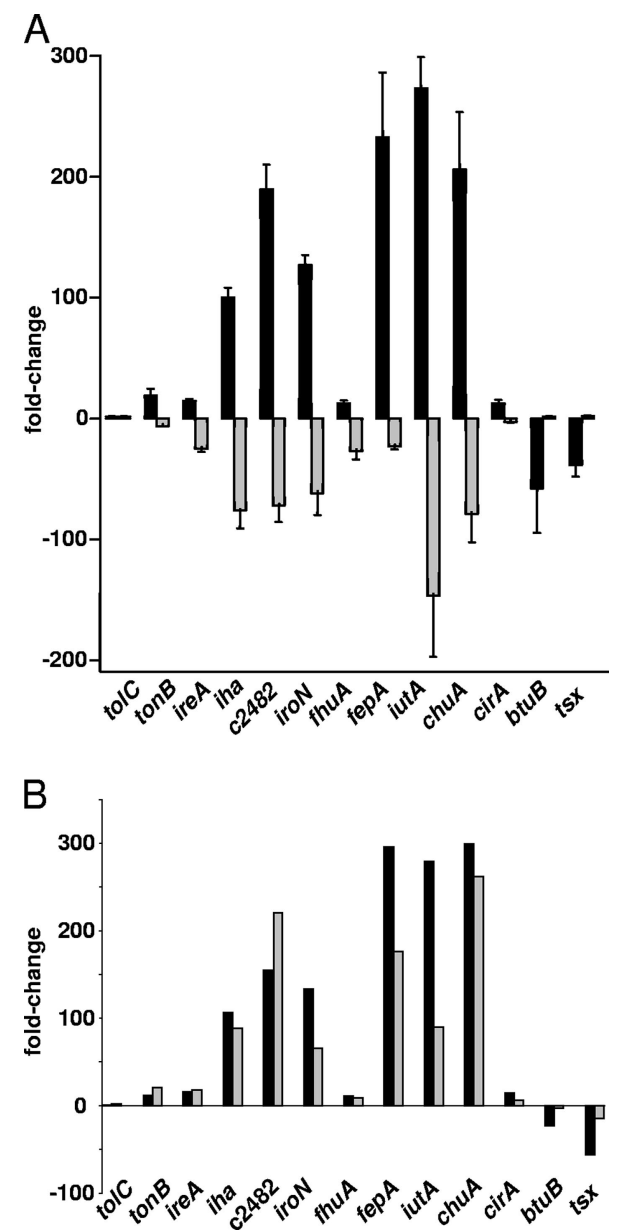

FIG. 6. qPCR for UPEC iron receptor genes during growth in urine, during growth in iron-replete urine, and during defined iron limitation. (A) qPCR results for CFT073 grown in human urine in the presence (gray bars) and absence (black bars) of $10 \mu \mathrm{M} \mathrm{FeCl}_{2}$ and (B) cultured in LB medium containing $10 \mathrm{mM}$ deferoxamine mesylate, an iron-chelating agent. Data were normalized to gap $A$ (glyceraldehyde 3-phosphate dehydrogenase) expression levels, and changes were determined by using LB medium expression levels as the calibrator. For iron-replete urine qPCR, urine without supplementation with ferrous chloride was used as the calibrator to generate $n$-fold change values. In panel $\mathrm{B}$, the black bars represent urine transcript $n$-fold changes and the gray bars represent LB-deferoxamine mesylate medium transcript levels compared to LB medium transcript levels.

iron compound receptors in urine is, in fact, dependent on iron limitation, as expected, the transcriptional analyses were extended to evaluate the production of the identified iron receptor mRNAs under iron-replete conditions. UPEC strain CFT073 was cultured in pooled human urine in the presence and absence of $10 \mu \mathrm{M} \mathrm{FeCl}_{2}$. This concentration of free iron did not affect the growth rate of the bacteria in urine (data not shown); however, the presence of $\mathrm{Fe}^{2+}$ substantially diminished the transcription of all of the iron compound receptor genes examined (Fig. 6A). The two most highly expressed receptor genes in urine, iutA and chuA, demonstrated the greatest decrease in transcription, down approximately 147.1and 79.1-fold, respectively (Fig. 6A) compared to their expression in urine with no added iron. A similar pattern of repres- 
sion was seen among the remaining six iron receptor genes during growth in human urine supplemented with ferrous chloride: iha, -76.4-fold; c2482, -72.1-fold; iroN, -69.1-fold; fhu $A$, -27.2-fold; ire $A,-25.2$-fold; fep $A,-23.5$-fold (Fig. 6A). Taken together, these data show that UPEC modulates the expression of these eight iron receptors in response to iron availability.

To further confirm that the observed induction of UPEC outer membrane receptors for iron in human urine is due to iron limitation, we used qPCR to examine the difference in the induction of the same genes in human urine and in LB medium containing $10 \mathrm{mM}$ deferoxamine mesylate, an iron-chelating agent (Fig. 6B). This concentration of deferoxamine mesylate reduces the growth rate of UPEC strain CFT073 in LB medium approximately threefold (data not shown). All but one gene, iut $A$, demonstrated a less-than-twofold difference between the level of transcription between urine and LB-deferoxamine mesylate medium. The induction of ire $A$ was 15.9 - and 17.4-fold greater than its expression in LB medium, human urine, and LB-deferoxamine mesylate medium, respectively. Similarly, the other $\operatorname{irg} A$ homolog, iha, was induced 107.0-fold in urine and 87.9-fold in LB-deferoxamine mesylate medium. The genes found to be downregulated in urine, $b t u B$ and $t s x$, also exhibited decreased expression in LB-deferoxamine mesylate medium. The $b t u B$ transcript level was found to be -3.2 -fold and the $t s x$ transcript level was -15 -fold in LB medium containing the iron chelator. Although there was a greater-than-twofold difference between urine and LB-deferoxamine mesylate medium, iutA was highly induced under both conditions, being 279.0-fold more highly expressed in urine and 90.4-fold more highly expressed in LB-deferoxamine mesylate medium than in LB medium. Because these iron receptor genes are highly expressed during culture in the presence of an iron-chelating agent and demonstrated significant reduction in transcription in iron-replete urine, the observed induction of these receptors in pooled human urine reflects the iron limitation encountered by UPEC in the urinary tract.

\section{DISCUSSION}

In this report, we describe the use of two proteomic advances, carbonate extraction of outer membrane proteins and 2D-DIGE, to profile and determine quantitative changes in the UPEC outer membrane subproteome during growth in human urine. Thirty individual outer membrane proteins were identified by $2 \mathrm{D}$ gel electrophoresis and MS/MS, and 12 of these proteins demonstrated significant differential expression between growth in human urine and that in LB medium. Of the 10 outer membrane proteins significantly induced in human urine, 7 (ChuA, IutA, FhuA, IroN, IreA, Iha, and c2482) are known or putative iron compound receptors. These findings show that human urine is an iron-limiting environment and concurrent production of numerous iron compound receptors by UPEC may represent a fundamental strategy for the ability of this pathogen to colonize the human urinary tract.

Since culturing UPEC in human urine partially mimics the urinary tract environment (42), we were interested in determining whether urine is capable of supporting the growth of other strains of $E$. coli. It has been known for nearly a century and a half that urine can serve as a growth medium for bacteria
(28), and it has been shown that urine supports the growth of both asymptomatic bacteriuria and UPEC strains $(3,38)$. It has also been suggested that growth in urine may be an important predictor of the colonization ability of uropathogens $(2,43)$. When the growth rates of select uropathogenic, intestinal pathogenic, and fecal-commensal $E$. coli isolates in human urine were examined, we found that nearly all of the isolates grew at similar rates in urine (Fig. 1A), with the exception of laboratory-adapted fecal-commensal E. coli strain MG1655 (K-12) (Fig. 1B). Further, although K-12 displayed an increased lag phase in relation to strain CFT073 $(8.43 \pm 0.68 \mathrm{~h}$, $P=0.0003)$, it too was eventually able to grow logarithmically at a rate similar to that of the other strains examined (Fig. 1B). Since we have shown that a number of $E$. coli stains, including the intestinal pathogens enteropathogenic and enterohemorrhagic $E$. coli and fecal-commensal isolates, can grow in human urine, it is plausible that the ability to grow in human urine, in and of itself, may not correlate with the ability to colonize the urinary tract. By pooling human urine from multiple donors, it is possible to limit batch-to-batch variability for the purpose of proteomic and transcriptional profiling; however, it remains possible that certain individual urine samples are incapable of supporting the observed growth rates of some E. coli strains, as has been seen in another study (9).

Despite finding that the ability to grow in pooled urine is not limited to uropathogens; examining gene expression for UPEC during culture in human urine has been useful in identifying novel UPEC genes that contribute to uropathogenesis, such as iroN and ire $A(36,37)$, and metabolic genes required for optimal growth in urine, i.e., gua $A$ and $\arg C$ (38). Further, global transcriptional analyses have shown that growth in human urine ex vivo partially mimics the in vivo urinary tract environment (42). Bacterial outer membrane proteins represent critical targets for the development of improved antimicrobials or protective vaccines against pathogens because these proteins are exposed on the bacterial surface. Therefore, profiling the UPEC outer membrane subproteome during growth in human urine not only aids in the elucidation of how UPEC might directly interact with its surrounding environment but also identifies potential candidates for the development of improved UTI therapies.

All of the proteomic analyses of outer membrane proteins have been conducted with $E$. coli K-12 cultured in LB medium. One study that pioneered the use of carbonate extraction and ASB-14 to efficiently solubilize and visualize the $E$. coli outer membrane identified 25 outer membrane proteins by using 2D-PAGE and mass spectrometry (23). More recently, sarcosine extraction was used to solubilize outer membrane proteins, including those that may be complexed and partition with inner membranes from $E$. coli, and the resulting proteomic analysis identified 31 outer membrane proteins (13). Here, for the first time, we define the profile of outer membrane proteins for an $E$. coli pathotype during growth in its natural environment. By using the carbonate extraction method, 2D gel electrophoresis, and mass spectrometry, we identified 30 outer membrane proteins for UPEC strain CFT073 cultured in human urine (Table 2). The number and classes of identified outer membrane proteins are consistent with the proteomic analyses of $E$. coli $\mathrm{K}-12$ and demonstrate that the UPEC outer 
membrane was isolated and sufficiently solubilized for quantitative analysis by 2D-DIGE.

The most striking finding elucidated by quantitative 2DDIGE is the induction of iron compound receptors by UPEC during growth in human urine (Table 3 ). Because iron is sequestered by the host, it is known that iron acquisition is a key feature that allows bacterial pathogens to successfully colonize a host (31). The induction of eight iron compound receptors by UPEC strain CFT073 during growth in human urine reflects the iron limitation encountered by the pathogen in this environment, which is in agreement with previous observations suggesting that urine and the urinary tract are, in fact, iron limited $(37,41,42,53)$. This is further supported by the phylogenetic distribution of iron compound receptors in E. coli UTI isolates, where there appears to be a greater prevalence of siderophore receptors in cystitis isolates than in pyelonephritis isolates (15), presumably reflecting the iron limitation we and others have observed in human urine.

The finding that UPEC strain CFT073 actively produces seven iron receptors in its outer membrane provides clear and compelling evidence that iron acquisition is required for UPEC virulence in the urinary tract. IutA, the aerobactin siderophore receptor, and $\mathrm{ChuA}$, the heme receptor, were the most highly induced iron receptors during growth in human urine (Table 3). Interestingly, CFT073 mutants with changes in the genes encoding these receptors were shown to be attenuated in experimental UTI (45). Similarly, Iha, a catecholate siderophore receptor and adhesin, and its homologous receptor IreA, which were also induced in human urine (Table 3), have been shown to contribute to urovirulence $(14,36)$. The fact that the transcription of these receptor genes is diminished in the presence of free iron (Fig. 6A) suggests that they are Fur regulated. In fact, it has been shown that the CFT073 iha promoter directly interacts with Fur and is highly expressed during iron limitation (29). Taken together, these results suggest that the newly identified c2482 putative iron compound receptor for UPEC strain CFT073, found to be induced in the 2D-DIGE experiments (Table 3), may also be Fur regulated and could play a role in the pathogenesis of UTI.

In this study, it was possible to visualize 104 protein spots by 2D-PAGE and identify 30 outer membrane proteins for UPEC strain CFT073 during growth in human urine. Interestingly, many of the proteins identified were present in multiply charged isoforms. This could represent a bona fide posttranslational modification such as phosphorylation or perhaps a modification that occurred during sample preparation such as deamidation. Although we have made no attempt to determine the nature of this charge variance, these multiply charged isoforms are commonly observed in outer membrane preparations visualized by 2D-PAGE $(16,20,23)$. In all, the ability of UPEC strain CFT073 to express at least eight outer membrane receptors for iron compounds demonstrates the critical importance of iron acquisition during successful colonization of the urinary tract environment. Further, these data regarding the induction of these receptors during growth in human urine highlight the facts that this environment is iron limiting and UPEC possesses unusual redundancy in its ability to acquire iron from this niche.

Uncomplicated UTI caused by UPEC are common community-acquired infections and represent a significant financial burden on the health care system. Therefore, it would be desirable to prevent these infections and reduce their impact on the health care system by developing a vaccine capable of generating a protective immune response against UPEC. Is has been speculated that such a vaccine candidate should be surface exposed (40) and, further, might be most efficacious as a polyvalent subunit vaccine (39). By characterizing the profile of UPEC outer membrane proteins during growth in human urine and identifying proteins that are specifically induced in this environment, we not only learn about how these pathogens are adapted to this iron-limited environment but have potentially identified numerous surface-exposed vaccine candidates. Thus, the combination of outer membrane proteomics and quantitative 2D-DIGE represents a powerful tool to examine bacterial pathogens in their natural environment.

\section{ACKNOWLEDGMENTS}

This work was supported in part by Public Health Service grants AI43363 and AI059722 from the National Institutes of Health.

\section{REFERENCES}

1. Alban, A., S. O. David, L. Bjorkesten, C. Andersson, E. Sloge, S. Lewis, and I. Currie. 2003. A novel experimental design for comparative two-dimensional gel analysis: two-dimensional difference gel electrophoresis incorporating a pooled internal standard. Proteomics 3:36-44.

2. Anderson, J. D., F. Eftekhar, M. Y. Aird, and J. Hammond. 1979. Role of bacterial growth rates in the epidemiology and pathogenesis of urinary infections in women. J. Clin. Microbiol. 10:766-771.

3. Asscher, A. W., M. Sussman, W. E. Waters, R. H. Davis, and S. Chick. 1966. Urine as a medium for bacterial growth. Lancet ii:1037-1041.

4. Busler, V. J., V. J. Torres, M. S. McClain, O. Tirado, D. B. Friedman, and T. L. Cover. 2006. Protein-protein interactions among Helicobacter pylori $\mathrm{Cag}$ proteins. J. Bacteriol. 188:4787-4800.

5. Connell, I., W. Agace, P. Klemm, M. Schembri, S. Marild, and C. Svanborg. 1996. Type 1 fimbrial expression enhances Escherichia coli virulence for the urinary tract. Proc. Natl. Acad. Sci. USA 93:9827-9832.

6. Cullen, P. A., D. A. Haake, and B. Adler. 2004. Outer membrane proteins of pathogenic spirochetes. FEMS Microbiol. Rev. 28:291-318.

7. Foxman, B., R. Barlow, H. D'Arcy, B. Gillespie, and J. D. Sobel. 2000 Urinary tract infection: self-reported incidence and associated costs. Ann. Epidemiol. 10:509-515.

8. Friedman, D. B., D. L. Stauff, G. Pishchany, C. W. Whitwell, V. J. Torres, and E. P. Skaar. 2006. Staphylococcus aureus redirects central metabolism to increase iron availability. PLoS Pathog. 2:e87.

9. Gordon, D. M., and M. A. Riley. 1992. A theoretical and experimental analysis of bacterial growth in the bladder. Mol. Microbiol. 6:555-562.

10. Grandi, G. 2001. Antibacterial vaccine design using genomics and proteomics. Trends Biotechnol. 19:181-188.

11. Guyer, D. M., S. Radulovic, F.-E. Jones, and H. L. T. Mobley. 2002. Sat, the secreted autotransporter toxin of uropathogenic Escherichia coli, is a vacuolating cytotoxin for bladder and kidney epithelial cells. Infect. Immun. 70:4539-4546.

12. Heimer, S. R., D. A. Rasko, C. V. Lockatell, D. E. Johnson, and H. L. T. Mobley. 2004. Autotransporter genes pic and tsh are associated with Escherichia coli strains that cause acute pyelonephritis and are expressed during urinary tract infection. Infect. Immun. 72:593-597.

13. Huang, C. Z., X. M. Lin, L. N. Wu, D. F. Zhang, D. Liu, S. Y. Wang, and X. X. Peng. 2006. Systematic identification of the subproteome of Escherichia coli cell envelope reveals the interaction network of membrane proteins and membrane-associated peripheral proteins. J. Proteome Res. 5:3268-3276.

14. Johnson, J. R., S. Jelacic, L. M. Schoening, C. Clabots, N. Shaikh, H. L. T. Mobley, and P. I. Tarr. 2005. The IrgA homologue adhesin Iha is an Escherichia coli virulence factor in murine urinary tract infection. Infect. Immun. 73:965-971.

15. Johnson, J. R., M. A. Kuskowski, A. Gajewski, S. Soto, J. P. Horcajada, M. T. Jimenez de Anta, and J. Vila. 2005. Extended virulence genotypes and phylogenetic background of Escherichia coli isolates from patients with cystitis, pyelonephritis, or prostatitis. J. Infect. Dis. 191:46-50.

16. Lai, E. M., U. Nair, N. D. Phadke, and J. R. Maddock. 2004. Proteomic screening and identification of differentially distributed membrane proteins in Escherichia coli. Mol. Microbiol. 52:1029-1044.

17. Levine, M. M., E. J. Bergquist, D. R. Nalin, D. H. Waterman, R. B. Hornick, C. R. Young, and S. Sotman. 1978. Escherichia coli strains that cause diarrhoea but do not produce heat-labile or heat-stable enterotoxins and are non-invasive. Lancet i:1119-1122. 
18. Lilley, K. S., and D. B. Friedman. 2004. All about DIGE: quantification technology for differential-display 2D-gel proteomics. Expert Rev. Proteomics 1:401-409.

19. Litwin, M. S., C. S. Saigal, E. M. Yano, C. Avila, S. A. Geschwind, J. M. Hanley, G. F. Joyce, R. Madison, J. Pace, S. M. Polich, and M. Wang. 2005 Urologic Diseases in America Project: analytical methods and principal findings. J. Urol. 173:933-937.

20. Lopez-Campistrous, A., P. Semchuk, L. Burke, T. Palmer-Stone, S. J. Brokx, G. Broderick, D. Bottorff, S. Bolch, J. H. Weiner, and M. J. Ellison. 2005. Localization, annotation, and comparison of the Escherichia coli K-12 proteome under two states of growth. Mol. Cell Proteomics 4:1205-1209.

21. McMillan, D. J., and G. S. Chhatwal. 2005. Prospects for a group A streptococcal vaccine. Curr. Opin. Mol. Ther. 7:11-16.

22. Mobley, H. L. T., D. M. Green, A. L. Trifillis, D. E. Johnson, G. R. Chippendale, C. V. Lockatell, B. D. Jones, and J. W. Warren. 1990. Pyelonephritogenic Escherichia coli and killing of cultured human renal proximal tubular epithelial cells: role of hemolysin in some strains. Infect. Immun. 58:12811289.

23. Molloy, M. P., B. R. Herbert, M. B. Slade, T. Rabilloud, A. S. Nouwens, K. L. Williams, and A. A. Gooley. 2000. Proteomic analysis of the Escherichia col outer membrane. Eur. J. Biochem. 267:2871-2881.

24. Molloy, M. P., B. R. Herbert, B. J. Walsh, M. I. Tyler, M. Traini, J. C Sanchez, D. F. Hochstrasser, K. L. Williams, and A. A. Gooley. 1998. Extraction of membrane proteins by differential solubilization for separation using two-dimensional gel electrophoresis. Electrophoresis 19:837-844.

25. Nouwens, A. S., S. J. Cordwell, M. R. Larsen, M. P. Molloy, M. Gillings, M. D. Willcox, and B. J. Walsh. 2000. Complementing genomics with proteomics: the membrane subproteome of Pseudomonas aeruginosa PAO1. Electrophoresis 21:3797-3809.

26. Odenbreit, S. 2005. Adherence properties of Helicobacter pylori: impact on pathogenesis and adaptation to the host. Int. J. Med. Microbiol. 295:317324.

27. Opal, S. M., A. S. Cross, P. Gemski, and L. W. Lyhte. 1990. Aerobactin and alpha-hemolysin as virulence determinants in Escherichia coli isolated from human blood, urine, and stool. J. Infect. Dis. 161:794-796.

28. Pasteur, L. 1863. Investigation into the role attributed to atmospheric oxygen gas in the destruction of animal and vegetable substances after death. C. R. Acad. Sci. 56:734-740

29. Rashid, R. A., P. I. Tarr, and S. L. Moseley. 2006. Expression of the Esch erichia coli IrgA homolog adhesin is regulated by the ferric uptake regulation protein. Microb. Pathog. 41:207-217.

30. Rathsam, C. R. E. Eaton, C. L. Simpson, G. V. Browne, V. A. Valova, D. W. Harty, and N. A. Jacques. 2005. Two-dimensional fluorescence difference gel electrophoretic analysis of Streptococcus mutans biofilms. J. Proteome Res. 4:2161-2173

31. Ratledge, C., and L. G. Dover. 2000. Iron metabolism in pathogenic bacteria. Annu. Rev. Microbiol. 54:881-941.

32. Riley, L. W., R. S. Remis, S. D. Helgerson, H. B. McGee, J. G. Wells, B. R. Davis, R. J. Hebert, E. S. Olcott, L. M. Johnson, N. T. Hargrett, P. A. Blake, and M. L. Cohen. 1983. Hemorrhagic colitis associated with a rare Escherichia coli serotype. N. Engl. J. Med. 308:681-685.

33. Rippere-Lampe, K. E., A. D. O'Brien, R. Conran, and H. A. Lockman. 2001. Mutation of the gene encoding cytotoxic necrotizing factor type 1 (CNF1) attenuates the virulence of uropathogenic Escherichia coli. Infect. Immun. 69:3954-3964.

34. Roberts, J. A., B. I. Marklund, D. Ilver, D. Haslam, M. B. Kaack, G. Baskin, M. Louis, R. Mollby, J. Winberg, and S. Normark. 1994. The Gal $(\alpha 1-4) \mathrm{Gal}$ specific tip adhesin of Escherichia coli P-fimbriae is needed for pyelonephritis to occur in the normal urinary tract. Proc. Natl. Acad. Sci. USA 91:11889 11893.

35. Roos, V., G. C. Ulett, M. A. Schembri, and P. Klemm. 2006. The asymptom- atic bacteriuria Escherichia coli strain 83972 outcompetes uropathogenic $E$. coli strains in human urine. Infect. Immun. 74:615-624.

36. Russo, T. A., U. B. Carlino, and J. R. Johnson. 2001. Identification of a new iron-regulated virulence gene, ire $A$, in an extraintestinal pathogenic isolate of Escherichia coli. Infect. Immun. 69:6209-6216.

37. Russo, T. A., U. B. Carlino, A. Mong, and S. T. Jodush. 1999. Identification of genes in an extraintestinal isolate of Escherichia coli with increased expression after exposure to human urine. Infect. Immun. 67:5306-5314.

38. Russo, T. A., S. T. Jodush, J. J. Brown, and J. R. Johnson. 1996. Identification of two previously unrecognized genes (guaA and $\arg C$ ) important for uropathogenesis. Mol. Microbiol. 22:217-229.

39. Russo, T. A., and J. R. Johnson. 2006. Extraintestinal isolates of Escherichia coli: identification and prospects for vaccine development. Expert Rev. Vaccines 5:45-54.

40. Russo, T. A., C. D. McFadden, U. B. Carlino-MacDonald, J. M. Beanan, T. J. Barnard, and J. R. Johnson. 2002. IroN functions as a siderophore receptor and is a urovirulence factor in an extraintestinal pathogenic isolate of Escherichia coli. Infect. Immun. 70:7156-7160.

41. Shand, G. H., H. Anwar, J. Kadurugamuwa, M. R. Brown, S. H. Silverman, and J. Melling. 1985. In vivo evidence that bacteria in urinary tract infection grow under iron-restricted conditions. Infect. Immun. 48:35-39.

42. Snyder, J. A., B. J. Haugen, E. L. Buckles, C. V. Lockatell, D. E. Johnson, M. S. Donnenberg, R. A. Welch, and H. L. T. Mobley. 2004. Transcriptome of uropathogenic Escherichia coli during urinary tract infection. Infect. Immun. 72:6373-6381.

43. Stamey, T. A., and G. Mihara. 1980. Observations on the growth of urethral and vaginal bacteria in sterile urine. J. Urol. 124:461-463.

44. Stapleton, A., S. Moseley, and W. E. Stamm. 1991. Urovirulence determinants in Escherichia coli isolates causing first-episode and recurrent cystitis in women. J. Infect. Dis. 163:773-779.

45. Torres, A. G., P. Redford, R. A. Welch, and S. M. Payne. 2001. TonBdependent systems of uropathogenic Escherichia coli: aerobactin and heme transport and TonB are required for virulence in the mouse. Infect. Immun. 69:6179-6185.

46. Twine, S. M., N. C. Mykytczuk, M. Petit, T. L. Tremblay, P. Lanthier, J. W. Conlan, and J. F. Kelly. 2005. Francisella tularensis proteome: low levels of ASB-14 facilitate the visualization of membrane proteins in total protein extracts. J. Proteome Res. 4:1848-1854.

47. Unlü, M., M. E. Morgan, and J. S. Minden. 1997. Difference gel electrophoresis: a single gel method for detecting changes in protein extracts. Electrophoresis 18:2071-2077.

48. van den Bosch, J. F., L. Emody, and I. Kétyi. 1982. Virulence of haemolytic strains of Escherichia coli in various animal models. FEMS Microbiol. Lett. 13:427-430.

49. Warren, J. W. 1996. Urinary tract infections: molecular pathogenesis and clinical management, 1st ed. ASM Press, Washington, DC.

50. Welch, R. A., V. Burland, G. Plunkett III, P. Redford, P. Roesch, D. Rasko, E. L. Buckles, S. R. Liou, A. Boutin, J. Hackett, D. Stroud, G. F. Mayhew, D. J. Rose, S. Zhou, D. C. Schwartz, N. T. Perna, H. L. T. Mobley, M. S. Donnenberg, and F. R. Blattner. 2002. Extensive mosaic structure revealed by the complete genome sequence of uropathogenic Escherichia coli. Proc. Natl. Acad. Sci. USA 99:17020-17024.

51. Welch, R. A., E. P. Dellinger, B. Minshew, and S. Falkow. 1981. Haemolysin contributes to virulence of extra-intestinal $E$. coli infections. Nature 294:665667.

52. Yan, J. X., A. T. Devenish, R. Wait, T. Stone, S. Lewis, and S. Fowler. 2002. Fluorescence two-dimensional difference gel electrophoresis and mass spectrometry based proteomic analysis of Escherichia coli. Proteomics 2:16821698.

53. Zhang, J. P., and S. Normark. 1996. Induction of gene expression in Escherichia coli after pilus-mediated adherence. Science 273:1234-1236.

Editor: A. D. O’Brien 